\title{
Intuitionistic Weak Arithmetic
}

\author{
Morteza Moniri \\ Institute for Studies in Theoretical Physics and Mathematics (IPM), \\ P.O. Box 19395-5746, Tehran, Iran \\ email: ezmoniri@ipm.ir
}

\begin{abstract}
We construct $\omega$-framed Kripke models of $i \forall_{1}$ and $i \Pi_{1}$ non of whose worlds satisfies $\forall x \exists y(x=2 y \vee x=2 y+1)$ and $\forall x, y \exists z \operatorname{Exp}(x, y, z)$ respectively. This will enable us to show that $i \forall_{1}$ does not prove $\neg \neg \forall x \exists y(x=2 y \vee x=2 y+1)$ and $i \Pi_{1}$ does not prove $\neg \neg \forall x, y \exists z \operatorname{Exp}(x, y, z)$. Therefore, $i \forall_{1} \nvdash \neg \neg l o p$ and $i \Pi_{1} \nvdash \neg \neg i \Sigma_{1}$. We also prove that $H A \nvdash l \Sigma_{1}$ and present some remarks about $i \Pi_{2}$.
\end{abstract}

2000 Mathematics Subject Classification: 03F30, 03F55, $03 \mathrm{H} 15$.

Key words and phrases: Fragments of Heyting Arithmetic, Kripke Models, exp.

\section{Preliminaries}

Following [W1], [AM], [MM], [M1] and [M2] this paper continues the study of some weak fragments of Heyting arithmetic and Kripke models of them.

We fix the language $L=\{+, \cdot,<, 0,1\}$ of arithmetic throughout the paper.

By open formulas we mean quantifier-free formulas. $(\exists x \leq t) \varphi$ is an abbreviation for $\exists x(x \leq t \wedge \varphi)$ and $(\forall x \leq t) \varphi$ is an abbreviation for $\forall x(x \leq t \rightarrow \varphi)$, where $t$ is a term not involving $x$. A formula is bounded if all quantifiers occurring in it are bounded, i.e., occur in a context as above. $\Sigma_{0}, \Pi_{0}$ or $\Delta_{0}$-formulas are bounded formulas. For $n \geq 0, \Sigma_{n+1^{-}}$ formulas have the form $(\exists \bar{x}) \varphi$ where $\varphi$ in $\Pi_{n}, \Pi_{n+1}$-formulas have the form $(\forall \bar{x}) \varphi$ where $\varphi$ in $\Sigma_{n}$.

The hierarchy of $\forall_{n}$-formulas and of $\exists_{n}$-formulas are defined similarly by changing bounded formulas to open formulas.

Heything arithmetic $H A$ and its fragments $\left(P A^{-}\right)^{i}$, iop $(=$ iopen $), \operatorname{lop}(=$ lopen $)$ and $i \Delta_{0}$ are the intuitionistic counterparts of first order Peano Arithmetic $P A$ and its fragments $P A^{-}, \operatorname{Iop}(=$ Iopen $), \operatorname{Lop}(=\operatorname{Lopen})$ and $I \Delta_{0}$. More generally for any set $\Gamma$ of formulas we will use notations such as $i \Gamma$ and $l \Gamma$ in the same manner. 
We use the usual terminology about Kripke structures as in [TD]. A formula $\varphi(\bar{x})$ is decidable in a Kripke model $\mathcal{K}$ whenever $\mathcal{K} \Vdash \forall \bar{x}(\varphi(\bar{x}) \vee \neg \varphi(\bar{x}))$.

For a set $T$ of sentences, $T^{i}$ and $T^{c}$ denote its intuitionistical and classical deductive closures.

Let $\neg \neg$ iop denote the intuitionistic theory axiomatized by $\left(P A^{-}\right)^{i}+\left\{\neg \neg I_{x} \varphi: \varphi\right.$ is open $\}$. The theories $\neg \neg i \forall_{1}$ and $\neg \neg l o p$ are defined similarly, by either replacing the class of open formulas by $\forall_{1}$-formulas or the induction scheme by LNP. Also, $\neg \neg i \Pi_{1}$ will stand for the intuitionistic theory axiomatized by $i \Delta_{0}+\left\{\neg \neg I_{x} \varphi: \varphi \in \Pi_{1}\right\}$.

Below we give three facts which we will use throughout the paper. The proofs are straightforward.

Fact $1 \mathrm{~A} \forall_{1}$ (resp. $\Pi_{1}$ )-formula is forced at a node $\alpha$ of a Kripke model of $\left(P A^{-}\right)^{i}$ (resp. $i \Delta_{0}$ ) if and only if it is satisfied in (the world attached to) $\alpha$ and any node above $\alpha$ if and only if it is satisfied in the union of the worlds in any (complete) path above $\alpha$.

Fact 2 Suppose that $\mathcal{K} \Vdash\left(P A^{-}\right)^{i}$ (resp. $\left.\mathcal{K} \Vdash i \Delta_{0}\right)$ and $\varphi \in \exists_{1}\left(\right.$ resp. $\left.\varphi \in \Sigma_{1}\right)$. Then for each $\alpha \in K$, we have:

$$
\alpha \Vdash \varphi \Leftrightarrow M_{\alpha} \vDash \varphi
$$

If $\psi \in \forall_{2}$ (resp. $\left.\psi \in \Pi_{2}\right)$ then:

$$
\alpha \Vdash \psi \Leftrightarrow \forall \beta \geq \alpha M_{\beta} \vDash \psi
$$

Fact 3 For a linear Kripke model deciding atomic (resp. bounded)-formulas to force $i \forall_{1}$ (resp. $i \Pi_{1}$ ), it is necessary and sufficient that the union of the worlds in any (complete) path in it satisfies $I \forall_{1}$ (resp. $\left.I \Pi_{1}\right)$.

Proof It was proved in [M2], using induction on formulas, that if $\alpha$ is a node in a linear Kripke model deciding atomic formulas and $\varphi$ is an $\exists$-free formula, then $\alpha \Vdash \varphi$ if and only if the union of the worlds above $\alpha$ satisfies $\varphi$. Using this the proof is straightforward. $\square$

\section{Constructing Kripke models of $i \forall_{1}+\neg A E O$ and $i \Pi_{1}+\neg \exp$}

In this section we prove two independence results for $i \forall_{1}$ and $i \Pi_{1}$.

Let $A E O$ be the sentence $\forall x \exists y(x=2 y \vee x=2 y+1)$. It was proved in [MM, 3.1] that, iop does not prove $\neg \neg A E O$. Here, using the same method, we show that even $i \forall_{1}$ does not prove $\neg \neg A E O$.

Proposition 1.1 There is an $\omega$-framed Kripke model of $i \forall_{1}$ which forces $\neg A E O$.

Proof: Method 1 We use a modified version of the proof of [MM, 3.1]. Indeed we prove that for any nonstandard model $M$ of $I \forall_{1}$ including an element $t$ infinitely many times divisible by 2 , there is an $\omega$-framed Kripke model of $i \forall_{1}$ with no worlds satisfying $A E O$ such that the union of its worlds is a countable submodel of $M$ satisfying $I \forall_{1}$. 
Let $\left(\psi_{n}\right)_{n \in \omega}$ be an enumeration of all universal $L$-formulas with a distinguished free variable. Each universal formula $\varphi\left(x_{1}, \cdots, x_{k}\right), k \geq 1$, occurs $k$-times in this enumeration.

Let $M \vDash I \forall_{1}$ and $t \in M$ has the above mentioned property. Put $M_{0}=\mathbb{Z}[t]^{\geq 0}$ and let $\bar{p}_{0,0}, \bar{p}_{0,1}, \cdots$ be a list of all tuples of parameters from $M_{0}$ (an enumeration of $M_{0}^{<\omega}$ ).

Fix any $k \geq 0$. Assume that for each $i \leq k$ a subsemiring $M_{i}$ of $M$ together with an enumeration $\left(\bar{p}_{i, j}\right)_{j \in \omega}$ of $M_{i}^{<\omega}$ is given. For each $0 \leq i, j, m \leq k$ with $i+j \leq k$, if $\bar{p}_{i, j}$ does not have the same arity as the non-distinguished free variables in $\psi_{m}$ or if $M_{i} \models \neg \psi_{m}\left(0, \bar{p}_{i, j}\right)$ or $M \models \forall x \psi_{m}\left(x, \bar{p}_{i, j}\right)$, where $x$ is the distinguished free variable in $\psi_{m}$, then let $s_{i, j, m}=0$. Otherwise, let $s_{i, j, m}$ be the least element in $M$ for which $M \models \neg \psi_{m}\left(s_{i, j, m}+1, \bar{p}_{i, j}\right)$ (note that $\left.I \forall_{1} \vdash L \exists_{1}\right)$. Suppose $\psi_{m}\left(s_{i, j, m}+1, \bar{p}_{i, j}\right)$ is $\forall \bar{y} \varphi_{m}\left(s_{i, j, m}+\right.$ $\left.1, \bar{p}_{i, j}, \bar{y}\right)$, where $\varphi_{m}$ is open. Let $\bar{t}_{i, j, m}$ be any tuple of elements of $M$ such that $M \models$ $\neg \varphi_{m}\left(s_{i, j, m}+1, \bar{p}_{i, j}, \bar{t}_{i, j, m}\right)$. Let $M_{k+1}=M_{k}\left[s_{i, j, m}, \bar{t}_{i, j, m}: 0 \leq i, j, m \leq k, i+j \leq k\right]^{\geq 0}$.

Consider the Kripke structure on frame $\omega$ with $M_{k}$ attached to node $k$. We want to show that for any $m, 0 \Vdash I_{x} \psi_{m}(x, \bar{y})$. Fix $i \geq 0$ and let $\bar{p}_{i, j} \in M_{i}$, of the same arity as the number of non-distinguished free variables in $\psi_{m}$, be arbitrary. We need to show $i \Vdash I_{x} \psi_{m}\left(x, \bar{p}_{i, j}\right)$. It is easy to see that $\neg \neg I_{x} \psi_{m}\left(x, \bar{p}_{i, j}\right) \vdash_{i} I_{x} \psi_{m}\left(x, \bar{p}_{i, j}\right)$ and so it suffices to prove the following claim:

Claim We have $i+j+m+1 \Vdash I_{x} \psi_{m}\left(x, \bar{p}_{i, j}\right)$.

Proof of the Claim In constructing $M_{i+j+m+1}$ from $M_{i+j+m}$, the formula $\psi_{m}\left(x, \bar{p}_{i, j}\right)$ receives attention. Using Fact 1 , one can show that if $M_{i} \models \neg \psi_{m}\left(0, \bar{p}_{i, j}\right)$ or $M \models$ $\forall x \psi_{m}\left(x, \bar{p}_{i, j}\right)$, then $i+j+m+1 \Vdash I_{x} \psi_{m}\left(x, \bar{p}_{i, j}\right)$. Otherwise, by construction and Fact 1 again, $i+j+m+1$ does not force the second conjunct of the antecedent of $I_{x} \psi_{m}\left(x, \bar{p}_{i, j}\right)$ and so forces $I_{x} \psi_{m}\left(x, \bar{p}_{i, j}\right)$. This establishes the claim.

As any finitely generated ring is Noetherian, one can show that each of the worlds in the Kripke model is a model of $\neg A E O$. Let us prove this. Assume for the purpose of a contradiction that some world models $A E O$. Put $t_{0}=t$ and $t_{l+1}=\frac{t_{l}}{2}$. The ascending chain of ideals $\left(t_{0}\right) \subseteq\left(t_{1}\right) \subseteq\left(t_{2}\right) \subseteq \cdots$ in the ring generated by that model must stop as, by Hilbert's basis theorem, every finitely generated ring is Noetherian. So, for some $n \in \mathbb{N}$ and some $g$ in that world, $0=(2 g-1) t$. But this is impossible as $2 g-1 \neq 0$ and $t$ is infinitely large. This contradiction shows that for some $i, t_{i+1}$ does not exist, i.e., $t_{i}$ is not divisible by 2. Since our world is supposed to be a model of $A E O$ it would follow that $t_{i}$ is odd, which is impossible because this world is a subring of $M$ in which $t_{i}$ is divisible by 2 .

Now since the sentence $A E O$ is $\forall_{2}$, the Kripke model will force $\neg A E O$ (Fact 2) and we will be done with the proposition.

Method 2 Let $M=\left\{p_{0}, p_{1}, p_{2}, \ldots\right\}$ be a countable nonstandard model of $I \forall_{1}$ with $t=p_{0} \in M$ as above. For each $i \geq 0$, put $M_{i}=\mathbb{Z}\left[p_{0}, \cdots, p_{i}\right]^{\geq 0}$. Let $\mathcal{K}$ be the obvious $\omega$-framed Kripke model. We have $\bigcup M_{i}=M \models I \forall_{1}$ and therefore by Fact $3, \mathcal{K} \Vdash i \forall_{1}$. 
Again, each node of $\mathcal{K}$ is finitely generated and so $\mathcal{K} \Vdash \neg A E O$.

An intuitionistic theory $T^{i}$ is said to be closed under the rule Double Negation Shift $D N S$ if whenever $T^{i} \vdash \forall \bar{x} \neg \neg \varphi$, then $T^{i} \vdash \neg \neg \forall \bar{x} \varphi$ for any formula $\varphi$.

Theorem 1.2 (i) The theory $i \forall_{1}$ is not closed under the rule $D N S\left(\exists_{1}\right)$ (the rule $D N S$ restricted to $\exists_{1}$-formulas).

(ii) $i \forall_{1} \nvdash \neg \neg l o p$.

Proof (i) By Iop $\vdash A E O$ and closure of iop under the negative translation we have $i o p \vdash \forall x \neg \neg \exists y(x=2 y \vee x=2 y+1)$, while the above proposition shows $i \forall_{1} \nvdash \neg \neg A E O$.

(ii) By the proof of [AM, Th. 1.4], Kripke models of lop are exactly Iop-normal Kripke structures and so lop $\vdash A E O$. $\square$

Now we consider the theory $i \Pi_{1}$. Recall Wehmeier's result, $i \Pi_{1} \nvdash \exp$, where exp is the $\Pi_{2}$ sentence which says the exponentiation function is total. His proof is based on constructing a two-node Kripke model of $i \Pi_{1}$ such that its root is not a model of $\exp$, see [W1, Lemma 10]. Here we prove a stronger independence result.

Proposition 1.3 There is an $\omega$-framed Kripke model of $i \Pi_{1}$ which forces $\neg \exp$.

Proof Let $M$ be a countable nonstandard model of $I \Pi_{1}$. Suppose that $a_{0}, a_{1}, a_{2}, \cdots$ is a cofinal sequence of the nonstandard elements of $M$ such that $a_{i}^{a_{i}}<a_{i+1}$ for each $i \geq 0$. For each $a \in M, a^{\mathbb{N}}$ denotes the set $\left\{x \in M: x<a^{n}\right.$ for some non negative integer $n\}$. Consider the Kripke Model $a_{0}{ }^{\mathbb{N}} \subseteq a_{1}{ }^{\mathbb{N}} \subseteq a_{2}{ }^{\mathbb{N}} \subseteq \cdots$. By [K, P. 69], each node of this Kripke model is a $\Delta_{o}$-elementary substructure of $M$ (therefore models $\Pi_{1}$-theory $I \Delta_{0}$ ) and non of them satisfies exp. Therefore, it forces the negation of exp $\in \Pi_{2}$. Also, since the union of the worlds in this Kripke model is equal to $M$ by Fact 3, it forces $i \Pi_{1}$.

Theorem 1.4 (i) The theory $i \Pi_{1}$ is not closed under the rule $\operatorname{DNS}\left(\Sigma_{1}\right)$ (the rule $D N S$ restricted to $\Sigma_{1}$-formulas).

(ii) $i \Pi_{1} \nvdash \neg \neg i \Sigma_{1}$.

Proof (i) The theory $i \Pi_{1}$ is closed under the negative translation and $I \Pi_{1}$ proves exp. Therefore $i \Pi_{1} \vdash \forall x, y \neg \neg \exists z E x p(x, y, z)$ while the above proposition shows $i \Pi_{1} \nvdash \neg \neg \exp$.

(ii) By [W1, Fact 8], $I \Sigma_{1}$ is $\Pi_{2}$-conservative over $i \Sigma_{1}$ and so $i \Sigma_{1} \vdash \exp$.

For any theory $T^{i}$ containing $i \Delta_{0}$, we denote the intuitionistic closure of $i \Delta_{0}+\{\neg \neg \varphi$ : $\left.\varphi \in T^{i}\right\}$ by $\neg \neg T^{i}$.

Proposition 1.5 If $T^{i}$ contains $i \Delta_{0}+\exp$, then $\neg \neg T^{i} \nvdash T^{i}$.

Proof Suppose $\neg \neg T^{i} \vdash T^{i}$. Then any two-node Kripke model consisting of a model $M \vDash T^{c}$ over a $\Delta_{0}$-elementary substructure of $M$ will force $T^{i}$, and so Whehmeier's argument about the limitation of the $\Pi_{2}$-consequences of $i \Pi_{1}$ works in this situation, contradiction. $\square$ 


\section{Some remarks about $i \Pi_{2}$}

What can we say about $i \Pi_{2}$ ? First, $I \Pi_{2}$ is $\Pi_{2}$-conservative over $i \Pi_{2}$ [Bur, Coro. 2.6]. Also, by Proposition 1.5, $\neg \neg i \Pi_{2} \nvdash i \Pi_{2}$. This shows that, unlike $i \Pi_{1}$, it is not true that satisfying $I \Pi_{2}$ in the union of each cofinal path of a Kripke model $\mathcal{K} \Vdash i \Delta_{0}$ implies $\mathcal{K} \Vdash i \Pi_{2}$. Therefore, we should not expect to construct Kripke models of the form Proposition 1.3 for $i \Pi_{2}$. However, the converse remains open:

Question 1 Is it true that the union of the worlds in any cofinal path of a Kripke model of $i \Pi_{2}$ satisfies $I \Pi_{2}$ ?

Wehmeier [W2, Th. 5.1] proved that any reversely well founded $I \Pi_{2}$-normal Kripke structure forces $i \Pi_{2}$ (note that by [Bus, P. 72-73], there exists an $\omega$-framed $P A$-normal Kripke structure which does not force even $\left.i \Pi_{1}\right)$. Also one can construct a non $I \Pi_{2}$-normal Kripke model of $i \Pi_{2}$ by putting a model $M$ of $I \Pi_{2}$ above a $\Sigma_{2}$-elementary subsructure of $M$ which is not a model of $I \Pi_{2}$. Furthermore, it is easy to see that any $\Sigma_{2}$-elementary $I \Pi_{2}$-normal Kripke structure forces $i \Pi_{2}$.

Question 2 Is there an $\omega$-framed Kripke model of $i \Pi_{2}$ non of whose worlds satisfies $I \Pi_{2}$ ?

Here we prove a generalization of [W2, Th. 5.1].

Proposition 2.1 Any $I \Pi_{2}$-normal Kripke model of $\neg \neg i \Pi_{2}$ (with a tree as its frame) forces $i \Pi_{2}$.

Proof Let $\mathcal{K}$ be an $I \Pi_{2}$-normal Kripke model of $\neg \neg i \Pi_{2}$ and $\alpha \in \mathcal{K}$. Suppose that $\varphi(x, \bar{y})$ is any $\Pi_{2}$-formula. If $\alpha \nVdash I_{x} \varphi(x, \bar{y})$, then there exists a node $\beta \geq \alpha$ and $\bar{b} \in M_{\beta}$ such that $\beta \Vdash \varphi(0, \bar{b})$ and $\beta \Vdash \forall x(\varphi(x, \bar{b}) \rightarrow \varphi(x+1, \bar{b}))$, but $\beta \nVdash \forall x \varphi(x, \bar{b})$. By $\beta \Vdash \neg \neg i \Pi_{2}$ in each path above $\beta$, there exists a node which forces $I_{x} \varphi(x, \bar{b})$ and so does $\forall x \varphi(x, \bar{b})$. Now we can consider the nodes below these nodes and proceed by bar induction as the proof of [W2, Th. 5.1].

We end this section by providing a proof for a stronger version of the fact $H A \nvdash L N P$, see e.g. [TD, P. 130-131] or [D, P. 117].

Proposition 2.2 $H A \nvdash l \Sigma_{1}$.

Proof Let $\tau \in \Pi_{1}$ be a Godel sentence $(P A \nvdash \tau, \mathbb{N} \models \tau)$. Assume $\sigma \equiv_{c} \neg \tau \in \Sigma_{1}$ and let $M$ be a classical model of $P A+\sigma$. Let $\mathcal{K}$ be the two-node Kripke model obtained by putting $M$ above $\mathbb{N}$ (the result of applying Smorynski's prime operation ' to $M[\mathrm{~S}]$ ). Note that the least solution of the formula $x=1 \vee \sigma$ in $\mathbb{N}$ is 1 and in $M$ is 0 . Hence using fact 2 , one can see that $\mathcal{K} \nVdash L_{x}(x=1 \vee \sigma)$.

Acknowlegements This research is supported by Institute for Studies in Theoretical 
Physics and Mathematics (IPM), Tehran, Iran.

\section{References}

[AM] M. Ardeshir and Mojtaba Moniri, Intuitionistic Open Induction and Open Least Number Principle and the Buss Operator, Notre Dame J. Formal Logic 39 (1998), $212-220$.

[Bur] W. Burr, Fragments of Heyting Arithmetic, J. Symbolic Logic 63 (2000), 1223-1240.

[Bus] S. Buss, Intuitionistic Validity in T-normal Kripke Structures, Ann. Pure Appl. Logic 59 (1993), 159-173.

[D] A. G. Dragalin, Mathematical intuitionism. Introduction to proof theory. Translated from the Russian by Elliott Mendelson. Translation edited by Ben Silver. Translations of Mathematical Monographs, 67. American Mathematical Society, Providence, RI, 1988.

[HP] P. Hajek and P. Pudlak, Metamathematics of First-order Arithmetic, SpringerVerlag, Berlin, 1993.

[K] R. Kaye, Models of Peano Arithmetic, Oxford University Press, Oxford, 1991.

[MM] Morteza Moniri and Mojtaba Moniri, Some Weak Fragments of HA and Certain Closure Properties, J. Symbolic Logic 67 (2002) 91-103.

[M1] Morteza Moniri, Weak Arithmetics and Kripke Models, Math. Logic Quart. 48 (2002) 157-160.

[M2] Morteza Moniri, Independence Results for Weak systems of Intuitionistic Arithmetic, Math. Logic Quart., to appear.

[S] C. Smorynski, Applications of Kripke Models, in: Metamathematical Investigations of Intuitionistic Arithmetic and Analysis (A.S. Troelstra, ed.), Springer Lecture Notes in Mathematics, Vol. 344, Springer-Verlag, Berlin, 1973.

[TD] A.S. Troelstra and D. van Dalen, Constructivism in Mathematics, Vol. I, NorthHolland, Amsterdam, 1988.

[W1] K.F. Wehmeier, Fragments of $H A$ Based on $\Sigma_{1}$-Induction, Arch. Math. Logic 37 (1997), 37-49.

[W2] K.F. Wehmeier, Constructing Kripke Models of Certain Fragments of Heyting's Arithmetic. Publ. Inst. Math. (Beograd) (N.S.) 77 (1998), 1-8. 